\title{
Multivariate approach to determine the ecological preferences of phytoplankton in Uppanar estuary, southeast coast of India
}

\author{
M. Saravanakumar, P. Murugesan, P. Damotharan and R. Punniyamoorthy \\ Centre of Advanced Study in Marine Biology, Annamalai University, Parangipettai, 608502, Tamil Nadu, India.
}

\section{To Cite this Article}

M. Saravanakumar, P. Murugesan, P. Damotharan and R. Punniyamoorthy. Multivariate approach to determine the ecological preferences of phytoplankton in Uppanar estuary, southeast coast of India. International Journal for Modern Trends in Science and Technology 2021, 7 pp. 23-33. https://doi.org/10.46501/IJMTST0710004

\section{Article Info}

Received: 30 August 2021; Accepted: 24 September 2021; Published: 29 September 2021

\section{ABSTRACT}

In the present study, the phytoplankton diversity and distribution was studied in relation seasonal variation of environmental parameters in the Uppanar estuary. Samples were seasonally collected (i.e., pre-monsoon: July-2018 and summer: June-2019) from six stations. A total of 62 phytoplankton species belonging to three classes, 20 orders, 26 families and 36 genera were and among the three phytoplankton taxa, diatoms topped the list with 41 species followed by Dinoflagellates (17 species) and blue green algae (4 species). The maximum number of phytoplankton species (36) and diversity value $\left(\mathrm{H}^{\prime}\right) 3.976$ was recorded in the stations near river mouth (UE-6) during summer and minimum species number (21) and maximum species richness (d) 6.923 was recorded in stations close to industrialr zone (UE-1) during monsoon. The maximum species evenness (J') 0.928 was recorded at stations near river mouth (UE-6). BIO-ENV (Biota-Environmental matching) and CCA results confirmed that the environmental parameters

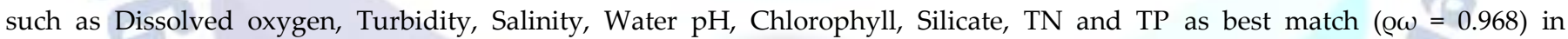
determining phytoplankton distributions. The results of present study helps to develop an understanding on the phytoplankton distribution based on physico-chemical parameters, which will form a reliable tool in bio-monitoring studies.

KEYWORDS: Phytoplankton, Environmental parameters, Density, Diversity, Multivariate, Uppanar estuary

\section{I.INTRODUCTION}

In any given aquatic environment, phytoplankton growth and abundance are largely regulated by both biotic and abiotic interactions [1]. Among abiotic interactions, the fresh water inflow influences greatly the abundance of planktonic organisms in marine ecosystem [2]. The constant nutrient supply always supports the rich phytoplankton production but generally nitrogen $(\mathrm{N})$ and phosphorous $(\mathrm{P})$ have been considered as the potentially limiting nutrients for phytoplankton growth in the aquatic ecosystems [3]. In addition to nutrients, physical properties such as salinity [4] turbidity and light availability [5, 6] are also known to play major roles in the regulation of phytoplankton growth and distribution.

Photosynthetic production by phytoplankton in euphotic layer supplies organic material and energy to the aquatic food chain and its spatial and seasonal variations affect the dynamics of material cycling in aquatic environments. The uptake of $\mathrm{PCO}_{2}$ by phytoplankton from the surface seawater for photosynthesis $[7,8]$ helps in carbon cycling. Phytoplankton distribution and abundance plays a central role in controlling the fact of biogenic carbon (C) in pelagic ecosystems [9]. High rates of primary production and organic matter re-mineralization along river-dominated coastal margins are largely the results 
of riverine inputs of terrestrially-derived dissolved and particulate materials [10].

The hydrodynamic pattern controls nutrient supply to the euphotic layer. High nutrient concentrations cause a preferential increase in the biomass and primary production [11, 12]. Based on fundamental knowledge on the phytoplankton species composition, density, and physiological state of organisms, it is possible to assess the degree of water pollution. It is important to know the probability and possible rate of water "self-purification" due to filtering and metabolic activities of planktonic organisms [13]. Phytoplankton responds within days to changes in light or nutrients and sediment load, and in response to grazing by larger zooplankton. In order to manage and maintain good water quality, we need to have thorough understanding on the phytoplankton and their interaction with the environment.

Therefore from environmental manager's perspective, the amount of phytoplankton in the water can inform about the ecological status of the aquatic ecosystem and wherein a management action is required. Accordingly, an attempt was made presently to study the abundance and diversity of phytoplankton in Uppanar estuary and the influence of seasonal variation on environmental parameters were also predicted to know the most influential abiotic factors, which determine the community structure of phytoplankton.

\section{MATERIALS AND METHODS}

\section{Study area}

Seasonal sampling was carried out from July 2018 June 2019 to catalogue the phytoplankton diversity and to determine the predominant environmental parameters, which influences the diversity and distribution of phytoplankton in Uppanar estuary. Six sampling stations were fixed (Fig. 1) and the details of sampling stations are given below:

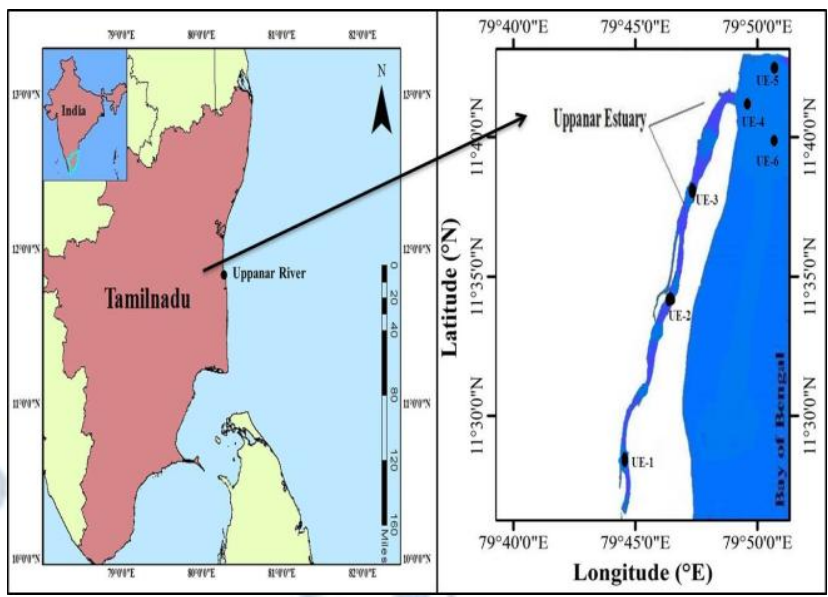

Fig. 1. Map showing the sampling stations in Uppanar estuary Station-1 UE-1 - Fixed near SPIC Pharma Industries (Lat. $11^{\circ} 38^{\prime} 47.77^{\prime \prime} \mathrm{N}$,

Long. 79²4'33.05"E)

Station-2 UE-2 - Fixed near Gradient zone

(Lat. $11^{\circ} 41^{\prime} 29.62^{\prime \prime} \mathrm{N}$,

Long. 79046'01.40"E)

Station-3 UE-3 - Fixed near urban discharge point (Lat. $11^{\circ} 40^{\prime} 17.12 " \mathrm{~N}$,

Long. 79045'27.13"E)

Station-4 UE-4 - Near Uppanar river mouth

(Lat. $11^{\circ} 42^{\prime} 15.73^{\prime \prime} \mathrm{N}$,

Long. $\left.79^{\circ} 47^{\prime} 43.73^{\prime \prime} \mathrm{E}\right)$

Station-5 UE-5 - 500m right side from Uppanar mouth (Lat. $11^{\circ} 40^{\prime} 58.51^{\prime \prime} \mathrm{N}$,

Long. $\left.79^{\circ} 47^{\prime} 21.66^{\prime \prime E}\right)$

Station-6 UE-6 - 500m left side from Uppanar mouth (Lat. $11^{\circ} 43^{\prime} 36.19^{\prime \prime} \mathrm{N}$,

Long. 7948'11.59"E)

Uppanar estuary which is formed by the confluence of Gadilam and Paravanar rivers and that opens in to Bay of Bengal near Cuddalore old town in the southeast coast of India. It is an open type estuary having the tidal effect extending up to a distance of about $6 \mathrm{~km}$ and average depth about $3.0 \mathrm{~m}$. The width of estuary is about $30 \mathrm{~m}$ near the mouth and $20 \mathrm{~m}$ in the upstream [14]. It runs behind the SIPCOT (State Industrial Promotion Corporation of Tamil Nadu Limited) industrial complex which consists of many chemical and pharmaceutical industries. The untreated effluents of these industries are directly discharged to this estuary. The navigational activities of indigenous fishing crafts, drainage of municipal and domestic sewage from the Cuddalore old town and new towns and wastes from coconut husk retaining grounds are also liquidated to this estuary. In addition to the above pollutants, the drainage channels 
from the nearby agriculture lands also discharges water with synthetic contaminants like pesticides and fertilizer into this estuary.

\section{Water sample analysis}

The physico-chemical parameters such as Temperature (Hand held thermometer), Salinity (Hand Refractometer - ATAGO Japan) and $\mathrm{pH}$ (pH pen- LI-120 Eutech Instrument Singapore) were recorded in situ by using the standard instruments and DO was estimated using Winkler's method as described by [15]. The water samples were collected in 1L polypropylene bottles by using a Niskin water sampler and Turbidity, Nitrite, Nitrate, Ammonia, Total Nitrogen (TN), Inorganic Phosphate (IP), Total Phosphorus (TP), Silicate (SiO3) were analysed by following the method described by [16]. Chlorophyll-a (Chl-a) were analyzed by spectrophotometric method by [15]. Total Suspended Solids (TSS) was determined by filtration and gravimetric technique [17]. The samples were analyzed in triplicates for physicochemical parameters and data quality was ensured through careful standardization and procedural blank measurements.

\section{Phytoplankton Samples}

The phytoplankton samples were collected at each station from surface water by horizontal hauling of phytoplankton net (mesh size of 25-50 $\mu \mathrm{m}$ ) for 5 minutes and preserved in 5\% formaldehyde. Phytoplankton samples were transported to the laboratory and kept for $48 \mathrm{~h}$. The supernatant was siphoned in order to make the sample denser and the remaining was centrifuged for several times for $5 \mathrm{~min}$ at $3000 \mathrm{rpm}$ to gain $30 \mathrm{ml}$ volume of each sample (Centrifuge Model Machine, R-8C, 8x15ml).

To identify phytoplankton species, each sample was shaken well to obtain a homogenized sample. Thereafter, three replicates of each sample $(1 \mathrm{ml})$ were pipetted from the $30 \mathrm{ml}$ sample [18, 19]. Subsequently, the organisms were stored, counted and identified up to group level using stereomicroscope (KL-300LED Carl Zeiss) up to lowest possible taxonomic level by consulting the standard works of $[20,21,19,22,23,24]$.

\section{Data analysis}

The data on environmental variables and biological variables were subjected to simple correlation and they were also treated with multivariate methods namely PCA (Pearson Correlation Analysis) and BIO-ENV (Biota-Environment matching) by using the statistical software PRIMER (Ver.7) (Plymouth Routines In Multivariate Ecological Research, Ver. 7.0) [25].

\section{RESULTS}

\section{Environmental data}

\section{Physico-chemical characteristics of water samples}

The mean and standard deviation (SD) of physico-chemical parameters of water and sediment are summarized in Table. 1. Water Depth range varied from $3.06 \pm 0.38$ to $13.38 \pm 0.65$

with minimum at UE-1 during summer and maximum at UE-5 during monsoon; Water temperature varied from $26.25 \pm 0.81$ to $28.75 \pm 1.03\left({ }^{\circ} \mathrm{C}\right)$ with minimum at UE-1 during monsoon and maximum at UE-5 during summer; water $\mathrm{pH}$ showed minimum of $7.76 \pm 0.49$ at UE-1 during monsoon and maximum of $8.13 \pm 1.05$ at UE-6 during summer; salinity showed a wide range of fluctuation with minimum $(18.44 \pm 1.52 \mathrm{ppt})$ at UE-1 during monsoon and maximum (35.5 $\pm 0.98 \mathrm{ppt})$ at UE-5 during summer. Dissolved oxygen ranged between 4.37 $\pm 0.85 \mathrm{mg} / \mathrm{l}$ at UE- 6 during monsoon and $3.49 \pm 1.08 \mathrm{mg} / \mathrm{l}$ at UE-1 during summer. Chlorophyll- $a$ varied from $2.586 \pm 0.37$ to $4.47 \pm 0.52 \mathrm{ppm}$ with the minimum value was recorded at UE-1 during summer and the maximum at UE-6 during monsoon. The Turbidity ranged between $4.58 \pm 0.95 \mathrm{NTU}$ at UE-5 during monsoon and $6.94 \pm 1.18$ NTU at UE-1 during post-monsoon. Particulate organic carbon content ranged from $80.73 \pm 1.84$ (UE-5 during summer) to $128.68 \pm 1.65 \mathrm{mgC} / \mathrm{l}$ (UE-1 during monsoon).

\section{Water Nutrients}

The Nitrite varied between $1.067 \pm 0.19$ at UE-1 during summer and $0.41 \pm 0.28$ at UE-5 during pre-monsoon. The Nitrate content ranged from $1.545 \pm$ 0.15 at UE-3 during summer and $4.79 \pm 0.35 \mu \mathrm{mol} / 1$ at UE-2 during monsoon. Ammonia content ranged from $0.04 \pm 0.005$ to $0.13 \pm 0.06 \mu \mathrm{mol} / 1$ and the maximum was recorded at UE-5 during monsoon and minimum at UE-3 during summer. Total Nitrogen varied between $14.35 \pm 1.69$ at UE-5 during summer and $23.78 \pm 2.81$ at UE-2 during monsoon. Inorganic phosphate varied between $1.32 \pm 0.22$ at UE-1 during monsoon and $0.41 \pm$ 0.10 at UE-6 during summer. Total Phosphate content ranged from $1.09 \pm 0.29$ to $2.68 \pm 0.25 \mu \mathrm{mol} / 1$ and the maximum was recorded at UE-6 during monsoon and minimum at UE-2 during summer. Silicates content varied from $20.39 \pm 0.68$ to $41.39 \pm 1.15 \mu \mathrm{mol} / \mathrm{l}$ and the 
Table 1. Physico-chemical characteristics (mean and SD) recorded in various sampling stations of the Uppanar estuary

\begin{tabular}{|c|c|c|c|c|c|c|}
\hline Variables & UE-1 & UE-2 & UE-3 & UE-4 & UE-5 & UE-6 \\
\hline Depth (m) & $3.06 \pm 0.38$ & $3.81 \pm 0.26$ & $2.56 \pm 0.41$ & $10.5 \pm 0.37$ & $13.38 \pm 0.65$ & $12.5 \pm 0.53$ \\
\hline W. Temp. $\left({ }^{\circ} \mathrm{C}\right)$ & $26.25 \pm 0.81$ & $27.38 \pm 1.07$ & $27.0 \pm 0.93$ & $28.0 \pm 1.56$ & $28.75 \pm 1.03$ & $28.63 \pm 0.94$ \\
\hline W. pH & $7.76 \pm 0.49$ & $7.83 \pm 0.28$ & $7.78 \pm 0.37$ & $7.95 \pm 0.71$ & $8.10 \pm 0.91$ & $8.13 \pm 1.05$ \\
\hline Salinity (ppt) & $18.44 \pm 1.52$ & $20.81 \pm 2.39$ & $24 \pm 1.24$ & $32.63 \pm 1.48$ & $35.5 \pm 0.98$ & $34.75 \pm 1.42$ \\
\hline DO (mg/l) & $3.49 \pm 1.08$ & $3.68 \pm 0.84$ & $3.59 \pm 0.73$ & $4.42 \pm 1.02$ & $4.29 \pm 0.79$ & $4.37 \pm 0.85$ \\
\hline Chl. a (ppm) & $2.586 \pm 0.37$ & $2.803 \pm 0.19$ & $3.257 \pm 0.38$ & $3.955 \pm 0.41$ & $2.75 \pm 0.53$ & $4.47 \pm 0.52$ \\
\hline Turbidity (NTU) & $6.94 \pm 1.18$ & $6.46 \pm 0.89$ & $5.86 \pm 0.91$ & $5.22 \pm 1.06$ & $4.58 \pm 0.95$ & $4.72 \pm 0.87$ \\
\hline POC (mgC/l) & $128.68 \pm 1.65$ & $113.03 \pm 2.19$ & $122.90 \pm 1.48$ & $81.49 \pm 2.53$ & $80.73 \pm 1.84$ & $84.21 \pm 1.52$ \\
\hline $\mathrm{NO}_{2}(\mu \mathrm{mol} / \mathrm{l})$ & $1.067 \pm 0.19$ & $0.86 \pm 0.24$ & $0.965 \pm 0.37$ & $0.78 \pm 0.19$ & $0.41 \pm 0.28$ & $0.44 \pm 0.11$ \\
\hline $\mathrm{NO}_{3}(\mu \mathrm{mol} / \mathrm{l})$ & $4.79 \pm 0.35$ & $4.52 \pm 0.16$ & $2.856 \pm 0.28$ & $1.257 \pm 0.11$ & $1.78 \pm 0.24$ & $1.545 \pm 0.15$ \\
\hline $\mathrm{NH}_{3}(\mu \mathrm{mol} / \mathrm{l})$ & $0.10 \pm 0.03$ & $0.10 \pm 0.05$ & $0.13 \pm 0.06$ & $0.05 \pm 0.004$ & $0.04 \pm 0.005$ & $0.06 \pm 0.003$ \\
\hline $\mathrm{TN}(\mu \mathrm{mol} / \mathrm{l})$ & $22.98 \pm 1.84$ & $23.78 \pm 2.81$ & $16.36 \pm 1.69$ & $15.41 \pm 1.82$ & $14.35 \pm 1.69$ & $15.99 \pm 0.97$ \\
\hline IP $(\mu \mathrm{mol} / 1)$ & $1.32 \pm 0.22$ & $1.26 \pm 0.14$ & $1.02 \pm 0.20$ & $0.60 \pm 0.28$ & $0.85 \pm 0.13$ & $0.41 \pm 0.10$ \\
\hline $\mathrm{TP}(\mu \mathrm{mol} / \mathrm{l})$ & $2.56 \pm 0.13$ & $2.68 \pm 0.25$ & $2.26 \pm 0.14$ & $2.31 \pm 0.16$ & $1.19 \pm 0.19$ & $1.09 \pm 0.29$ \\
\hline $\mathrm{SiO}_{3}(\mu \mathrm{mol} / \mathrm{l})$ & $22.26 \pm 0.84$ & $20.39 \pm 0.68$ & $25.59 \pm 1.57$ & $38.23 \pm 1.49$ & $41.28 \pm 1.08$ & $41.39 \pm 1.15$ \\
\hline
\end{tabular}

(Footnote: W. Temp - Water Temperature; W. pH - Water pH; DO - Dissolved Oxygen; Chl.a - Chlorophyll; $\mathrm{SiO}_{3}-$ Silicate; $\mathbf{N O}_{2}$ - Nitrite; $\mathbf{N O}_{3}$ - Nitrate; $\mathbf{N H}_{3}$ - Ammonia; $\mathbf{T N}$ - Total Nitrogen; $\mathbf{T P}$ - Total Phosphate; IP - Inorganic Phosphate; POC - Particulate Organic Carbon)

\section{Biological characteristics}

\section{Phytoplankton}

From the above surveyed stations, a total of 62 phytoplankton species belonging to three groups (Blue-green algae, diatom and dinoflagellates), 20 orders, 26 families and 36 genera were identified from the Uppanar estuary. Of these, diatoms were found to be the dominant group with 41 species (Table 2). Dinoflagellates formed next dominant group with 17 species and blue green algae came last in the order with 4 species.

Sampling stations in industrial zone (UE-1, UE-2 and UE-3) phytoplankton species such as Asterionella glacialis, Bellerochea malleus, Chaetoceros affinis, C. debilis, Coscinodiscus grani, Guinardia delicatula, Ceratium bucephalum, C. furca, Dinophysis punctata, Triceratium favus, Peridinium diabolus, Anabeana sp. and Tricodesmium erythraeum were found commonly and besides the phytoplankton species such as Lithodesmium undulatum, Melosira sp., Navicula hennedyii, Odontella sinensis, Pleurosigma normanii, Planktonella sol, Rhizosolenia crassispina and Thalassionema nitzschioides, Protoperidinium depressum, Pyrophagus stenii and Trichodesmium sp. were recorded abundantly in coastal stations (UE-4, UE-5 and UE-6). Among the seasons, the maximum number (36 species) of plankton species was recorded at stations near coastal zone (UE-5 and UE-6) during summer and minimum (21 species) was recorded at stations near industrial zone (UE-1 and UE-2) during monsoon.

Phytoplankton population density varied from 4564 to 9894 Cells/1 with maximum was recorded at UE-5 during summer and minimum at UE-1 during monsoon (Fig. 2). 


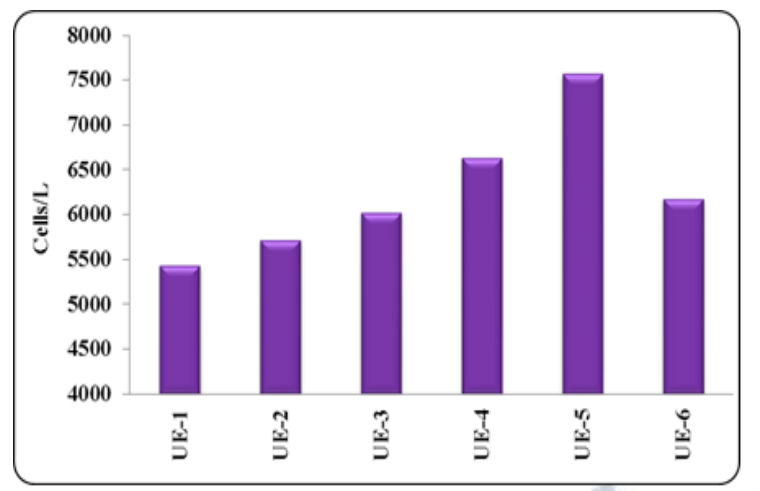

Fig. 2. Population density of phytoplankton recorded in various stations of Uppanar estuary

\subsection{Percentage contribution}

The results of percentage composition of phytoplankton revealed that diatoms constituted the maximum with $75 \%$ of the total followed by dinoflagellates with $16 \%$ and blue greens with $9 \%$ of the total percentage composition of community (Fig. $3)$.

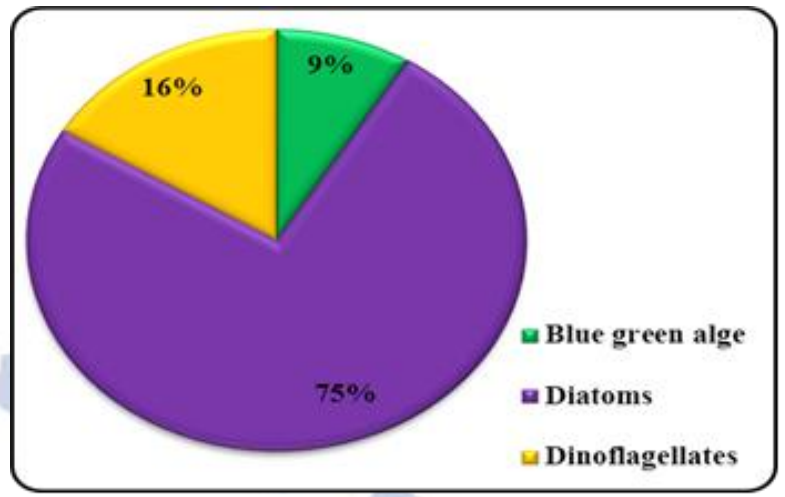

Fig. 3. Percentage contribution of phytoplankton orders recorded in various sampling stations of the Uppanar estuary

Table 2. Taxonomical classification of phytoplankton recorded from Uppanar estuary

\begin{tabular}{|c|c|c|c|c|}
\hline Class & Order & Family & Genus & Phytoplankton Species \\
\hline Blue-green algae & Oscillatoriales & Oscillatoriaceae & Lyngbya & Lyngbya sp. C.Agardh ex Gomont, 1892 \\
\hline & & 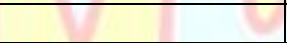 & Oscillatoria & Oscillatoria sp.Vaucher ex Gomont, 1892 \\
\hline & & Phormidiaceae & Trichodesmium & $\begin{array}{l}\text { Trichodesmium erythraeum Ehrenberg ex } \\
\text { Gomont, } 1892\end{array}$ \\
\hline$\frac{1}{76}$ & +2 & & & $\begin{array}{l}\text { Trichodesmium thiebautii Gomont ex } \\
\text { Gomont, } \mathbf{1 8 9 0}\end{array}$ \\
\hline \multirow[t]{5}{*}{ Diatom } & Fragilariales & Fragilariaceae & Asterionella & Asterionella glacialis Castracane, 1886 \\
\hline & Bacillariales & Bacillariaceae & Bacillaria & Bacillaria paradoxa J.F.Gmelin, 1791 \\
\hline & 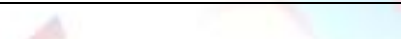 & & Nitzschia & Nitzschia closterium Allen \& Nelson, 1910 \\
\hline & & & & Nitzschia longissima Allen \& Nelson, 1910 \\
\hline & & 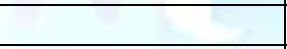 & & Nitzschia seriata Allen \& Nelson, 1910 \\
\hline 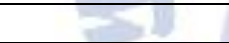 & Chaetocerotanae incertae sedis & Chaetocerotaceae & Bacteriastrum & Bacteriastrum comosum J.Pavillard, 1916 \\
\hline & & & & Bacteriastrum hyalinum Lauder, 1864 \\
\hline 20 & & & Chaetoceros & Chaetoceros affinis Lauder, 1864 \\
\hline & & & & Chaetoceros curvisetus Cleve, 1889 \\
\hline & & & & Chaetoceros diversus Cleve, $\mathbf{1 8 8 9}$ \\
\hline & & & & Chaetoceros indicus Cleve, 1889 \\
\hline & Hemiaulales & Bellerocheaceae & Bellerochea & Bellerochea malleus Van Heurck, 1885 \\
\hline & P & Hemiaulaceae & Hemiaulus & Hemiaulus sinensis Greville, 1865 \\
\hline & +2 & & & Hemidiscus hardmanianus Greville, 1865 \\
\hline & & & Lauderia & Lauderia borealis Gran, 1900 \\
\hline & Biddulphiales & Biddulphiaceae & Biddulphia & $\begin{array}{l}\text { Biddulphia heteroceros Grunow in Van } \\
\text { Heurck, } \mathbf{1 8 8 2}\end{array}$ \\
\hline & 1 & & Streptotheca & $\begin{array}{l}\text { Streptotheca thamensis W.H. Shrubsole, } \\
\mathbf{1 8 9 0}\end{array}$ \\
\hline & Hemiaulales & Hemiaulaceae & Climacodium & Climacodium frauenfeldianum Grunow, 1868 \\
\hline & Hemiaulales & +1 & Eucampia & Eucampia zodiacus Ehrenberg, 1839 \\
\hline & Coscinodiscales & Coscinodiscaceae & Coscinodiscus & Coscinodiscus centralis Ehrenberg, 1844 \\
\hline & & & & Coscinodiscus gigas Ehrenberg, 1844 \\
\hline & & & & Coscinodiscus radiates Ehrenberg, 1844 \\
\hline & Thalassiosirales & Stephanodiscaceae & Cyclotella & Cyclotella sp. A. de Brébisson, 1838 \\
\hline & & Skeletonemaceae & Skeletonema & Skeletonema costatum Cleve, $\mathbf{1 8 7 3}$ \\
\hline & & & Thalassiosira & Thalassiosira sp. Manguin, 1957 \\
\hline & & Thalassiosiraceae & Planktoniella & Planktoniella sol Schütt, 1892 \\
\hline & Rhizosoleniales & Rhizosoleniaceae & Guinardia & Guinardia flaccida H.Peragallo, 1892 \\
\hline & & & Rhizosolenia & Rhizosolenia alata Brightwell, 1858 \\
\hline & & & & Rhizosolenia imbricate Brightwell, 1858 \\
\hline
\end{tabular}




\begin{tabular}{|c|c|c|c|c|}
\hline & & & & Rhizosolenia pungens Brightwell, 1858 \\
\hline & & & & Rhizosolenia setigera Brightwell, 1858 \\
\hline & & & & Rhizosolenia styliformis Brightwell, 1858 \\
\hline & Leptocylindrales & Leptocylindraceae & Leptocylindrus & Leptocylindrus danicus Cleve, $\mathbf{1 8 8 9}$ \\
\hline & Licmophorales & Licmophoraceae & Licmophora & Licmophora abbreviate C.Agardh, 1831 \\
\hline & Triceratiales & Triceratiaceae & Odontella & Odontella mobiliensis Grunow, $\mathbf{1 8 8 4}$ \\
\hline & & & & Odontella sinensis Grunow, $\mathbf{1 8 8 4}$ \\
\hline & & & Triceratium & Triceratium favus Ehrenberg, 1839 \\
\hline & & & & Triceratium reticulatum Greville, 1865 \\
\hline & Naviculales & Pleurosigmataceae & Pleurosigma & Pleurosigma directum Grunow, $\mathbf{1 8 8 0}$ \\
\hline & & & & Pleurosigma normanii Grunow, $\mathbf{1 8 8 0}$ \\
\hline & Melosirales & Stephanopyxidaceae & Stephanopyxis & Stephanopyxis palmeriana Grunow, $\mathbf{1 8 8 4}$ \\
\hline & Thalassionematales & Thalassionemataceae & Thalassiothrix & Thalassiothrix frauenfeldii Grunow, $\mathbf{1 8 8 0}$ \\
\hline & 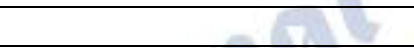 & 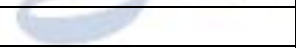 & $\log ^{2}$ & Thalassiothrix nitzschioides Grunow, $\mathbf{1 8 8 1}$ \\
\hline & Lithodesmiales & Lithodesmiaceae & Ditylum & Ditylum brightwellii Grunow, $\mathbf{1 8 8 5}$ \\
\hline Dinflagellates & Gonyaulacales & Ceratiaceae & Ceratium & $\begin{array}{l}\text { Ceratium furca Claparède \& Lachmann, } \\
1859\end{array}$ \\
\hline & +4 & & & Ceratium fusus Dujardin, 1841 \\
\hline & 20 & 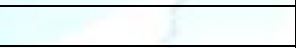 & 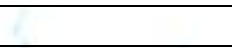 & Ceratium inflatum E.G.Jørgensen, 1911 \\
\hline & +4 & 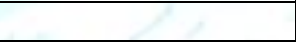 & 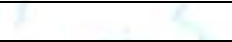 & Ceratium macroceros Cleve, 1899 \\
\hline & +1 & - & 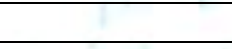 & Ceratium pulchellum Cleve, $\mathbf{1 8 9 9}$ \\
\hline 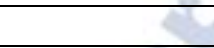 & 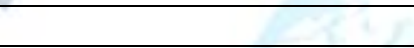 & & 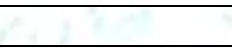 & Ceratium trichoceros Schröder, 1906 \\
\hline 6 & 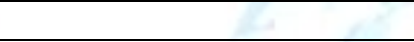 & & & Ceratium tripos Nitzsch, $\mathbf{1 8 1 7}$ \\
\hline 30 & Dinophysiales & Dinophysaceae & Dinophysis & Dinophysis caudate Saville-Kent, $\mathbf{1 8 8 1}$ \\
\hline+2 & & & & Dinophysis punctata Jörgensen, 1923 \\
\hline 5 & Peridiniales & Peridiniaceae & Peridinium & Peridinium sp. Cleve, 1900 \\
\hline+2 & +2 & 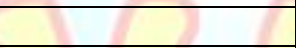 & (2) & Peridinium diabolus Cleve, 1900 \\
\hline & +2 & Protoperidiniaceae & Protoperidinium & Protoperidinium oceanicum Balech, 1974 \\
\hline 2 & Prorocentrales & Prorocentraceae & Prorocentrum & Prorocentrum micans Ehrenberg, $\mathbf{1 8 3 4}$ \\
\hline 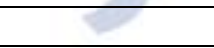 & Gonyaulacales & Pyrophacaceae & Pyrophacus & Pyrophacus steinii Wall \& Dale, 1971 \\
\hline
\end{tabular}

\section{Diversity indices}

The Shannon diversity $\left(\mathrm{H}^{\prime}\right)$ index calculated for phytoplankton abundance data showed minimum (2.541) at UE-1 during monsoon and maximum (3.972) at UE-6 during summer season; Margalef species richness (d) showed minimum (5.018) at UE-6 during pre-monsoon and maximum (6.923) at UE-1 in

Table 3. Diversity indices, a-Shannon diversity $\left(\mathrm{H}^{\prime}\right)$; b-Margalef richness (d) c- Pielou's evenness ( $\left.\mathrm{J}^{\prime}\right)$ and d-Simpson dominance (D) calculated for phytoplankton Uppanar estuary

\begin{tabular}{|l||l|l||l||l||}
\hline Stations & Shannon diversity $\left.\mathbf{( H}^{\prime}\right)$ & Margalef richness (d) & Pielou's evenness (J') & $\begin{array}{l}\text { Simpson } \\
\text { Dominance (D) }\end{array}$ \\
\hline \hline UE-1 & 2.541 & 6.923 & 0.741 & 0.908 \\
\hline UE-2 & 2.957 & 6.918 & 0.794 & 0.875 \\
\hline \hline UE-3 & 2.946 & 6.276 & 0.776 & 0.849 \\
\hline \hline UE-4 & 3.068 & 6.475 & 0.816 & 0.835 \\
\hline \hline UE-5 & 3.161 & 5.713 & 0.823 & 0.578 \\
\hline \hline UE-6 & 3.972 & 5.018 & 0.928 & 0.664 \\
\hline
\end{tabular}

\section{Cluster/MDS Analysis}

Further, to study the similarity/dissimilarity between stations, phytoplankton abundance of six different stations were approached to cluster analysis and MDS (non-metric Multi-Dimensional Scaling) ordination (Fig. 4). The dendrogram showed that the summer; Pielou's species evenness ( $\mathrm{J}$ ') varied between 0.741 and 0.928 with maximum at UE-6 during summer and minimum at UE-1 during monsoon and Simpson dominance varied from 0.578 to 0.908 with maximum at UE-1 during Summer season and minimum at UE-5 in monsoon (Table. 3). 
overlying on the top-right corner of the MDS plot is also very minimal (0.05), signaling a good ordination pattern of phytoplankton abundance (Fig. 5).

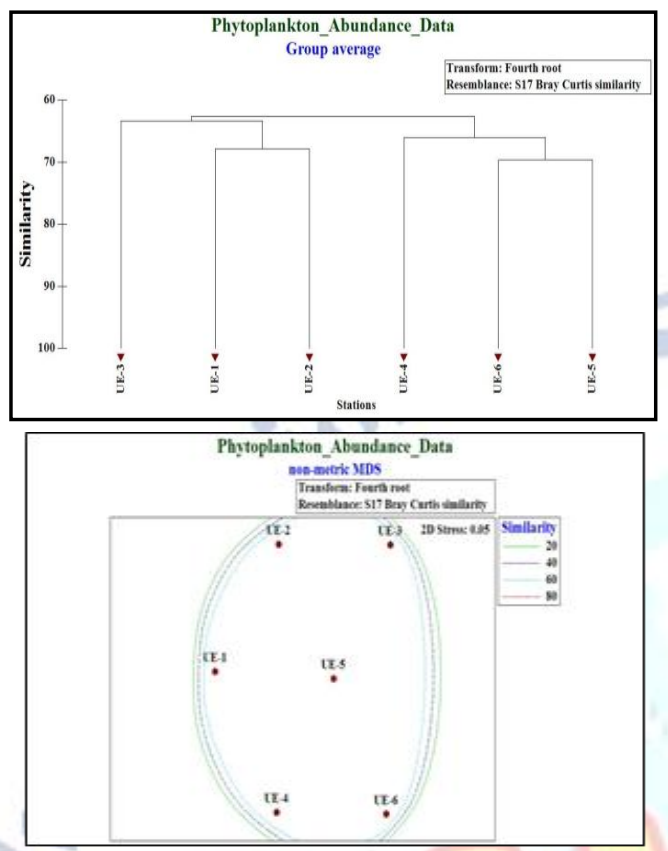

Fig. 4. Dendrogram and MDS plot drawn for the phytoplankton species data collected in various stations of Uppanar estuary

\section{BIO-ENV (Biota-Environment matching)}

In the BIO-ENV procedure, which was employed to measure the agreement between the rank correlations of the biological (Bray-Curtis similarity) and environmental (Euclidean distance) matrices, thirteen environmental variables (Temperature, Dissolved oxygen, Salinity, Water $\mathrm{pH}$, Turbidity, Chlorophyll, Silicate, Nitrite, Nitrate, Total Nitrogen, Inorganic Phosphate, Total Phosphate and Particulate organic carbon) were allowed to match the biota. Among the above parameters, a combination of eight environmental parameters namely Dissolved oxygen, Turbidity, Salinity, Water pH, Chlorophyll, Silicate, $\mathrm{TN}$ and TP got manifested as best match ( $\mathrm{p} \omega=0.968)$ in determination of phytoplankton distributions. Dissolved oxygen, Temperature, Salinity, $\mathrm{pH}$, Chlorophyll, Inorganic phosphate, and particulate organic carbon formed the next best ( $p \omega=0.931$ ) combination of environmental parameters (Table 4).

Table 4. Harmonic rank correlations $(\rho \omega)$ between phytoplankton abundance and environmental Similarity matrices in various stations of Uppanar estuary

\begin{tabular}{clc}
\hline No. of variables & \multicolumn{1}{c}{ Best variable combinations } & Correlation $(\rho \omega)$ \\
\hline 8 & DO - Turbidity - Salinity - W. PH - Chlorophyll - Silicate - TN - TP & 0.968 \\
\hline 7 & DO - Salinity - W. pH - Temperature - Chlorophyll - IP - POC & 0.931 \\
6 & Silicate - TSS - Ammonium - Nitrite - Nitrate - POC & 0.906 \\
5 & DO - Chlorophyll - W. pH - Salinity - Silicate & 0.842 \\
4 & Silicate - Temperature - Salinity - Chlorophyll & 0.749 \\
\hline
\end{tabular}

\section{Pearson-Correlation matrix}

Further, Pearson correlation (r) was also done to ascertain correlation between the Physico-chemical characteristics and phytoplankton diversity components (Table 5). The correlation matrix revealed positive significant correlation $(\mathrm{P}<0.001)$ for the following combination of parameters: Diversity vs Evenness (0.996); $\mathrm{TN}$ vs $\mathrm{SiO}_{3}$ (0.991); Total phosphate vs Inorganic Phosphate (0.980); $\mathrm{SiO}_{3}$ vs Diversity (0.984); W. pH vs DO (0.956); Salinity vs W. $\mathrm{pH}$ (0.947); Chlorophyll vs $\mathrm{SiO}_{3}$ (0.910) Besides, a significant negative correlations $(\mathrm{P}<0.05)$ were found between parameters such as Diversity vs Richness (-0.993); POC vs Richness (- 0.990); Turbidity vs TN (-0.943); DO vs POC (-0.989); Chlorophyll vs Richness $(-0.952)$ and Salinity vs $\mathrm{NH}_{3}(-0.982)$ in various stations of Uppanar estuary (Table 3). 
Table 5. Correlation matrix between the physico chemical characteristics in different stations of Uppanar estuary

\begin{tabular}{|c|c|c|c|c|c|c|c|c|c|c|c|c|c|c|c|c|c|}
\hline Parameters & Temp & Sali & W. pH & DO & Chl. a & Turb & $\mathrm{NO}_{2}$ & $\mathrm{NO}_{3}$ & $\mathrm{NH}_{3}$ & TN & TP & IP & $\mathrm{SiO}_{3}$ & POC & $\left(\mathrm{H}^{\prime}\right)$ & (d) & $\left(\mathrm{J}^{\prime}\right)$ \\
\hline W. Tem & 1 & & & & & & & & & & & & & & & & \\
\hline Salinity & -0.846 & 1 & & & & & & & & & & & & & & & \\
\hline W.pH & -0.836 & 0.947 & 1 & & & & & & & & & & & & & & \\
\hline $\mathrm{DO}$ & -0.785 & 0.814 & 0.956 & 1 & & & & & & & & & & & & & \\
\hline Chl. a & 0.478 & $\begin{array}{c}-0.67 \\
0\end{array}$ & -0.396 & $\begin{array}{c}-0.71 \\
6\end{array}$ & 1 & & & & & & & & & & & & \\
\hline Turbidity & 0.531 & $\begin{array}{c}-0.85 \\
0\end{array}$ & -0.660 & $\begin{array}{c}-0.41 \\
4\end{array}$ & -0.826 & 1 & & & & & & & & & & & \\
\hline $\mathrm{NO}_{2}$ & 0.632 & $\begin{array}{c}-0.84 \\
0\end{array}$ & -0.713 & $\begin{array}{c}-0.56 \\
5\end{array}$ & 0.747 & $\begin{array}{c}-0.70 \\
4\end{array}$ & 1 & & & & & & & & & & \\
\hline $\mathrm{NO}_{3}$ & 0.799 & $\begin{array}{c}-0.97 \\
6\end{array}$ & -0.854 & $\begin{array}{c}-0.66 \\
8\end{array}$ & 0.816 & $\begin{array}{c}-0.93 \\
0\end{array}$ & $\begin{array}{c}0.77 \\
4\end{array}$ & 1 & & & & & & & & & \\
\hline $\mathrm{NH}_{3}$ & 0.787 & $\begin{array}{c}-0.98 \\
2\end{array}$ & -0.871 & $\begin{array}{c}0.69 \\
1\end{array}$ & 0.795 & $\begin{array}{c}-0.78 \\
6\end{array}$ & $\begin{array}{c}0.65 \\
1\end{array}$ & $\begin{array}{c}0.67 \\
3\end{array}$ & 1 & & & & & & & & \\
\hline $\mathrm{TN}$ & 0.750 & $\begin{array}{c}-0.97 \\
7\end{array}$ & -0.867 & $\begin{array}{c}-0.68 \\
3\end{array}$ & 0.792 & $\begin{array}{c}-0.94 \\
3\end{array}$ & $\begin{array}{c}0.91 \\
7\end{array}$ & $\begin{array}{c}0.79 \\
4\end{array}$ & $\begin{array}{c}0.59 \\
8\end{array}$ & 1 & & & & & & & \\
\hline $\mathrm{TP}$ & 0.706 & $\begin{array}{c}-0.98 \\
0\end{array}$ & -0.896 & $\begin{array}{c}-0.75 \\
0\end{array}$ & 0.730 & $\begin{array}{c}-0.83 \\
5\end{array}$ & $\begin{array}{c}0.73 \\
0\end{array}$ & $\begin{array}{c}0.67 \\
8\end{array}$ & $\begin{array}{c}0.67 \\
4\end{array}$ & $\begin{array}{c}0.95 \\
8\end{array}$ & 1 & & & & & & \\
\hline IP & 0.812 & $\begin{array}{c}-0.93 \\
6 \\
\end{array}$ & -0.878 & $\begin{array}{c}-0.76 \\
9\end{array}$ & 0.642 & $\begin{array}{c}-0.71 \\
5\end{array}$ & $\begin{array}{c}0.76 \\
1 \\
\end{array}$ & $\begin{array}{c}0.71 \\
6\end{array}$ & $\begin{array}{c}0.70 \\
9\end{array}$ & $\begin{array}{c}0.88 \\
2 \\
\end{array}$ & $\begin{array}{c}0.98 \\
0 \\
\end{array}$ & 1 & & & & & \\
\hline $\mathrm{SiO}_{3}$ & 0.892 & $\begin{array}{c}-0.87 \\
7\end{array}$ & -0.727 & $\begin{array}{c}0.55 \\
0\end{array}$ & 0.910 & $\begin{array}{c}-0.79 \\
8\end{array}$ & $\begin{array}{c}0.72 \\
4\end{array}$ & $\begin{array}{c}0.72 \\
5\end{array}$ & $\begin{array}{c}-0.94 \\
8\end{array}$ & $\begin{array}{c}0.99 \\
1\end{array}$ & $\begin{array}{c}0.65 \\
3\end{array}$ & $\begin{array}{c}0.95 \\
4\end{array}$ & 1 & & & & \\
\hline POC & 0.774 & $\begin{array}{c}-0.76 \\
0\end{array}$ & -0.819 & $\begin{array}{c}-0.98 \\
9\end{array}$ & -0.851 & $\begin{array}{c}0.51 \\
7\end{array}$ & $\begin{array}{c}0.77 \\
2\end{array}$ & $\begin{array}{c}0.69 \\
8\end{array}$ & $\begin{array}{c}0.89 \\
5\end{array}$ & $\begin{array}{c}0.87 \\
8\end{array}$ & $\begin{array}{c}0.76 \\
7\end{array}$ & $\begin{array}{c}0.90 \\
1\end{array}$ & $\begin{array}{c}0.72 \\
7\end{array}$ & 1 & & & \\
\hline$\left(\mathrm{H}^{\prime}\right)$ & 0.748 & $\begin{array}{c}-0.72 \\
5\end{array}$ & -0.827 & $\begin{array}{c}0.68 \\
7\end{array}$ & 0.727 & $\begin{array}{c}-0.76 \\
2\end{array}$ & $\begin{array}{c}0.78 \\
3\end{array}$ & $\begin{array}{c}0.73 \\
4\end{array}$ & $\begin{array}{c}-0.72 \\
0\end{array}$ & $\begin{array}{c}0.89 \\
3\end{array}$ & $\begin{array}{c}0.78 \\
1\end{array}$ & $\begin{array}{c}0.79 \\
2\end{array}$ & $\begin{array}{c}0.98 \\
4\end{array}$ & $\begin{array}{c}0.62 \\
5\end{array}$ & 1 & 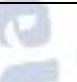 & \\
\hline (d) & 0.750 & $\begin{array}{c}-0.87 \\
7\end{array}$ & -0.867 & $\begin{array}{c}-0.68 \\
3\end{array}$ & -0.952 & $\begin{array}{c}-0.94 \\
3\end{array}$ & $\begin{array}{c}0.81 \\
7\end{array}$ & $\begin{array}{c}0.69 \\
4\end{array}$ & $\begin{array}{c}0.69 \\
8\end{array}$ & $\begin{array}{c}0.89 \\
3\end{array}$ & $\begin{array}{c}0.75 \\
0\end{array}$ & $\begin{array}{c}0.84 \\
3\end{array}$ & $\begin{array}{c}0.71 \\
7\end{array}$ & $\begin{array}{c}-0.99 \\
0\end{array}$ & $\begin{array}{c}-0.99 \\
3\end{array}$ & 1 & \\
\hline$\left(\mathrm{J}^{\prime}\right)$ & 0.706 & $\begin{array}{c}-0.68 \\
0\end{array}$ & -0.896 & $\begin{array}{c}0.75 \\
0\end{array}$ & 0.730 & $\begin{array}{c}-0.83 \\
5\end{array}$ & $\begin{array}{c}0.74 \\
8\end{array}$ & $\begin{array}{c}0.87 \\
8\end{array}$ & $\begin{array}{c}-0.77 \\
4\end{array}$ & $\begin{array}{c}0.71 \\
8\end{array}$ & $\begin{array}{c}0.80 \\
6\end{array}$ & $\begin{array}{c}0.63 \\
5\end{array}$ & $\begin{array}{c}0.83 \\
0\end{array}$ & $\begin{array}{c}0.84 \\
8\end{array}$ & $\begin{array}{c}0.68 \\
4\end{array}$ & $\begin{array}{c}0.73 \\
0\end{array}$ & 1 \\
\hline
\end{tabular}

\section{*P $<0.05$ significant correlation}

\section{** $\mathrm{P}<0.01$ strong significant correlation}

\section{***P $<0.001$ very strong significant correlation}

(Footnote: Temp - Water Temperature; Sali - Salinity W. pH - Water pH; DO - Dissolved Oxygen; Chl. a Chlorophyll; $\mathbf{N O}_{2}$ - Nitrite; $\mathbf{N O}_{3}$ - Nitrate; $\mathbf{N H}_{3}$ - Ammonia; $\mathbf{T N}$ - Total Nitrogen; TP - Total Phosphate; IP Inorganic Phosphate; $\mathrm{SiO}_{3}$ - Silicate; POC - Particulate organic carbon; (H') - Diversity; (d) - Richness and (J') Evenness)

\section{DISCUSSION}

Phytoplankton studies are useful for identification of the physico-chemical and other biological conditions of the water in any aquatic ecosystem. Some groups of phytoplankton can hamper recreational value of surface water, particularly by forming thick surface scum, which reduces the use of amenities for water sports or large growth, which cause de-oxygenation of the water leading to fish death [26]. Over the last few decades, there has been more concern about the processes influencing the development of phytoplankton communities, primarily in relation to physico-chemical factors $[27,28]$.
In the present study, the phytoplankton community comprised of diatoms, dinoflagellates, blue green algae at Uppanar estuary. Diatoms were the most dominant group in all the stations throughout the study period, which could be ascribed to the fact that diatoms could thrive well in varying environmental changes as reported earlier by several researchers $[29,30,31,32,33$, $34,35,36,37$ were studied in West coast]. Diatoms were followed by dinoflagellates, whereas blue green algae were represented by very few species during the study period. [35] Also observed the predominance of Bacillariopycean (diatoms) members followed by Chlorophyceae (blue greens) and Cyanophyceae (green algae) members during his study on phytoplankton 
community of Brazilian lakes, which lends supports to the results of the present study.

In estuarine environment, phytoplankton assemblage, structure and growth are affected by the different environmental factors that include salinity, nutrients, temperature etc. [38]. Phytoplankton distribution and their growth depend on several environmental factors, which are varied with seasons and regions. In Uppanar estuary, maximum density of phytoplankton was recorded during summer followed by post-monsoon and minimum density was during monsoon. The maximum density of phytoplankton could be attributed to the neritic element domination and availability of nutrients. As the hydrological parameters were in stable condition during postmonsoon season, that might have favored to record more phytoplankton species [39. 32]. Besides, more number of phytoplankton during postmonsoon could be due to increased radiation or light intensity [29, 33] noticed higher density of phytoplankton during post-monsoon months and lower in monsoon on Kaduviyar estuary, India, which corroborated well with the results of present investigation.

In the present study, a total of 62 species, three groups, 20 orders, 26 families and 36 genera were recorded. Number of species recorded in this study is fairly comparable with [40] who reported 77 species from Mahanadi estuary. The higher abundance and species diversity during summer and postmonsoon seasons might be due to the predominance of diatoms viz: Asterionella glacialis, Bellerochea malleus, Chaetoceros affinis, C. debilis, Coscinodiscus grani, Guinardia delicatula, Ceratium bucephalum, C. furca, Dinophysis punctata, Triceratium favus, Peridinium diabolus, Anabeana sp., Tricodesmium erythraeum, Lithodesmium undulatum, Melosira sp., Navicula hennedyii, Odontella sinensis, Pleurosigma normanii, Planktonella sol, Rhizosolenia crassispina, Thalassionema nitzschioides, Protoperidinium depressum and Pyrophagus stenii.

The higher phytoplankton abundance during summer and postmonsoon season could be attributed to the increased salinity, $\mathrm{pH}$, high temperature and high intensity of light penetration. Similar summer maxima and monsoonal minimal was reported earlier by [32]. The abundance was lowest during monsoon season, when the water column was remarkably stratified to a large extent because of heavy rainfall, high turbidity caused by run-off, reduced salinity, decreased temperature and $\mathrm{pH}$, overcast sky and cool conditions. However, during this season, freshwater algal forms like Oscillatoria sp., Lynbya sp. and Trichodesmium erythraeum were noticed. Similar observations have been made from different locations of east and west coast of India [41, 34, 42, 43, 44, 36] also reported that fresh water forms such as green algae and blue green algae.

With regard to diversity and density values, the maximum was recorded during summer and postmonsoon seasons and minimum during monsoon season. The high density and diversity recorded during summer could be attributed to more stable hydrographical conditions prevailed during that period. Similar observations were earlier made by [44, 30, 39, 32, 33]. Similarly, the richness values minimum during monsoon were reported earlier by [45] from Kollidam estuary, [46] from the Arasalar estuaries and [30] from Coleroon estuary. In the present study, cluster analysis revealed that the stations near coastal zone and stations near industrial zone were grouped to form a separate cluster based on species composition and abundance. Further, this was confirmed through MDS plot. This might be due to the difference in selection of stations formed separate grouping as evidenced by [47] in Kerala back waters. The dendrograms derived in the present study, showed major dichotomy in the clustering of samples collected in different stations, which agree well with [48] in Kalpakkam coastal waters

\section{CONCLUSION}

Based on the foregoing account, it is concluded that the present study yielded quite a good amount of information on the phytoplankton assemblage in Uppanar estuary, south east coast of India. The present study also reported as many as 62 phytoplankton species and of these, group diatom were found dominantly. The diversity indices values confirmed that the stations near industrial zone in Uppanar estuary were having less phytoplankton species variety and whereas the stations near coastal zone was having more diverse phytoplankton species assemblage.

Doubtless, the results of the present study would certainly supplement to the existing knowledge on seasonal variation of phytoplankton assemblages in tropical estuaries. The facts observed in this study were encouraging and it promotes the use of phytoplankton 
abundance based biotic indices as a bio-monitoring tool to ascertain the health of tropical aquatic ecosystem.

\section{Acknowledgements}

Authors are grateful to the Director \& Dean, CAS in Marine Biology \& authorities of Annamalai University for providing necessary facilities.

\section{Conflict of interest statement}

Authors declare that they do not have any conflict of interest.

\section{REFERENCES}

[1] Sin, Y. and Wetzel, R.L., 2002. Ecosystem modeling analysis of size-structured phytoplankton dynamics in the York River estuary, Virginia (USA). II. Use of a plankton ecosystem model for investigating controlling factors on phytoplankton and nutrient dynamics. Marine Ecology Progress Series, 228, pp.91-101.

[2] Cloern, J.E., 1996. Phytoplankton bloom dynamics in coastal ecosystems: a review with some general lessons from sustained investigation of San Francisco Bay, California. Reviews of Geophysics, 34(2), pp.127-168.

[3] Neill, M., 2005. A method to determine which nutrient is limiting for plant growth in estuarine waters at any salinity. Marine Pollution Bulletin 50: 945-955.

[4] Mc Lusky, D.S. and Heard, V.E.J., 1971. Some effects of salinity on the mysid Praunus flexuosus. Journal of the Marine Biological Association of the United Kingdom, 51(3), pp.709-715.

[5] Cloern, J.E., 1987. Turbidity as a control on phytoplankton biomass and productivity in estuaries. Continental Shelf Research 7: 1367-1381.

[6] Cloern, J.E., Foster, S.Q. and Kleckner, A.E., 2014. Phytoplankton primary production in the world's estuarine-coastal ecosystems. Biogeosciences, 11(9), pp.2477-2501.

[7] Watson, A.J., Robinson, C., Robinson, J.E., Williams, P.L.B. and Fasham, M.J.R., 1991. Spatial variability in the sink for atmospheric carbon dioxide in the North Atlantic. Nature, 350(6313), pp.50-53.

[8] Robertson, A.I. and Blaber, S.J.M., 1993. Plankton, epibenthos and fish communities. Coastal and estuarine studies, pp.173-173.

[9] Legendre, L. and Rassoulzadegan, F., 1996. Food-web mediated export of biogenic carbon in oceans: hydrodynamic control. Marine Ecology Progress Series, 145, pp.179-193.

[10] Dagg, M.J., Ammerman, J.W., Amon, R.M., Gardner, W.S., Green, R.E. and Lohrenz, S.E., 2007. A review of water column processes influencing hypoxia in the northern Gulf of Mexico. Estuaries and Coasts, 30(5), pp.735-752.

[11] Chisholm, S.W., 1992. Phytoplankton size. In Primary productivity and biogeochemical cycles in the sea (pp. 213-237). Springer, Boston, MA.

[12] Agawin, N.S., Duarte, C.M. and Agustí, S., 2000. Nutrient and temperature control of the contribution of picoplankton to phytoplankton biomass and production. Limnology and oceanography, 45(3), pp.591-600.

[13] Nelson, D.M., Tréguer, P., Brzezinski, M.A., Leynaert, A. and Quéguiner, B., 1995. Production and dissolution of biogenic silica in the ocean: revised global estimates, comparison with regional data and relationship to biogenic sedimentation. Global biogeochemical cycles, 9(3), pp.359-372.

[14] Karunagaran, V.M., 1990. Impact of wastewater from SIPCOT industrial complex on the water quality of Uppanar estuary (Lat. $11043^{\prime} \mathrm{N}$; Long $79049^{\prime} \mathrm{E}$ ) with special reference to fluoride contamination. M. Phil. Thesis, Annamalai University, India, pp 74

[15] Strickland, J.D.H., Parsons, T.R., 1972. A Practical Handbook of Seawater Analysis. 2nd edn. Fisheries Research Board of Canada Bull. 167 Alger Press, 310 pp.

[16] Grasshoff, K., Kremling, K. and Ehrhardt, M., 1999. Methods of Seawater Analysis Wiley.

[17] APHA, 1998. Standard Methods for the Examination of Water and Waste Water. 20th edition. American Public Health Association, Washington D.C., USA, 2.25 -4. 134.

[18] Vollenweider, R.A., Munawar, M. and Stadelmann, P., 1974. A comparative review of phytoplankton and primary production in the Laurentian Great Lakes. Journal of the Fisheries Board of Canada, 31(5), pp.739-762.

[19] Newell, G.E. and Newell, R.C., 1977. Marine plankton. Hutchinson.

[20] Prescott, D.M. and Bender, M.A., 1962. Synthesis of RNA and protein during mitosis in mammalian tissue culture cells. Experimental cell research, 26(2), pp.260-268.

[21] Tiffany, L.H. and Britton, M.E., 1971. Algae of illinois.

[22] Todd, C.D., Laverack, M.S. and Boxshall, G., 1996. Coastal marine zooplankton: a practical manual for students. Cambridge University Press.

[23] Perumal, P., Sampathkumar, P. and Karuppasamy, P.K., 1999. Studies on the bloom-forming species of phytoplankton in the Vellar estuary, southeast coast of India.

[24] Kasymov, A.G., 2004. Ecology of plankton in the Caspian Sea. Baku: Publ. house Adiloglu, ExxonMobil, Exxon Azerbaijan Operating Company I, I, C, an Exxon Mobil Subsidiary, p.542.

[25] Clarke, K.R. and Gorley, R.N., 2015. Getting started with PRIMER v7. PRIMER-E: Plymouth, Plymouth Marine Laboratory, 20.

[26] Whitton, B.A. and Patts, M. 2000. The Ecology of Cyanobacteria. Kluwer Academic Publishers, Netherlands.

[27] Peerapornpisal, Y., Sonthichai, W., Somdee, T., Mulsin, P. and Rott, E. 1999. Water quality and phytoplankton in the Mae Kuang Udomtara Reservoir, Chiang Mai, Thailand. J. Sci. Fac. Cmu., 26(1): 25-43.

[28] Elliott, J.A., Irish, A.E. and Reynolds, C.S. 2002. Predicting the spatial dominance of phytoplankton in light limited and incompletely mixed eutrophic water column using the PROTECH model. Fresh. Bio., 47: 433-440.

[29] Mani, P. 1992. Natural phytoplankton communities in Pichavaram mangroves, Indian J. mar. Sci., 12: 278- 280.

[30] Rajasegar, M., Srinivasan, M. and Rajaram, R., 2000. Phytoplankton diversity associated with the shrimp farm development in Vellar estuary, south India. Seaweed Res. Utiln, 22(1-2), pp.125-213. 
[31] Saravanakumar, A., J. Seshserebiah, G. A. Thivakaran and M. Rajkumar, 2007. Benthic macrofaunal assemblage in the arid zone mangroves of Gulf of Kutch, Gujatat, J. Ocean. Uni., China, 6: 33-39.

[32] Rajkumar, M., Perumal, P., Ashok Prabu, V., Vengadesh Perumal, N. and Thillai Rajasekar, K., 2009. Phytoplankton diversity in Pichavaram mangrove waters from south-east coast of India.

[33] Perumal, N.V., Rajkumar, M., Perumal, P. and Rajasekar, K.T., 2009. Seasonal variations of plankton diversity in the Kaduviyar estuary, Nagapattinam, southeast coast of India. J. Environ. Biol, 30(6), pp.1035-1046.

[34] Joseph, K. J. and V. K. Pillai, 1975. Seasonal and spatial distribution of phytoplankters in Cochin Backwaters. Bulletin Department of Marine Science, University, Cochin, 7: 171-180.

[35] Joy, C. M., K. P. Balakrishnan and A. Joseph, 1990. Effect of industrial discharges on the ecology of phytoplankton production in the River Periyar (India). Water Res., 24(6): 787-796.

[36] Madhu, N.V., Jyothibabu, R. and Balachandran, K.K., 2010. Monsoon-induced changes in the size-fractionated phytoplankton biomass and production rate in the estuarine and coastal waters of southwest coast of India. Environmental monitoring and assessment, 166(1), pp.521-528.

[37] Madhu, N.V., R. Jyothibabu, K. K. Balachandran, U. K. Honey, G. D. Martin, J. G. Vijay, C. A. Shiyas, G. V. M. Guptha and C. T. Achuthankutty, 2007. Monsoonal impact on planktonic standing stock and abundance in tropical estuary, Cochin back waters, India, Estuar. Coast. Shelf Sci., 73: 54-64.

[38] Gasiunaite, Z. R., A. C. Cardoso, A. S. Heiskanen, P. Henriksen, P. Kauppila, I. Olenina, R. Pikaityte, I. Purina, A. Razinkovas, S. Sagert, S. Schubert and N. Wasmund, 2005. Seasonality of coastal phytoplankton in the Baltic sea: influence of salinity and eutrophication. Estuar. Coast. Shelf Sci., 65: 239-252.

[39] Rajasekar, K.T., Peramal, P. and Santhanam, P., 2005. Phytoplankton diversity in the Coleroon estuary, Southeast coast of India. Journal of Marine biological association of India, 47, pp.127-132.

[40] Naik, S., B. C. Acharya and A. Mohapatra, 2009. Seasonal variations of phytoplankton in Mahanadi estuary, east coast of India. Indian J. Mar. Sci., 38: 184-190.

[41] Gopinathan, C. P., 1975. On new distributional records of plankton diatoms from the Indian Seas. J. Mar. Biol. Ass. India.17: 223-240.

[42] Murugan, A. and K. Ayyakkannu, 1993. Studies on the ecology of phytoplankton in Cuddalore Uppanar backwater, Southeast coast of India, Indian J. Mar. Sci., 22: 135-137.

[43] De, T. K., A. Choudhury and T. K. Jana, 1994. Phytoplankton community organization and species diversity in the Hoogly estuary, north east coast of India, Indian J. Mar. Sci., 23: 152-156.

[44] Gouda, R. \& Panigrahy R. C. (1996). Ecology of phytoplankton in coastal waters off Gopalpur, East coast of India. Indian J. Mar. Sci., 25: 81-84.

[45] Edward, J.P. and Ayyakkannu, K., 1991. Studies on the ecology of plankton community of Kollidam Estuary, Southeast Coast of India: 1. Phytoplankton. Mahasagar, 24(2), pp.89-97.

[46] Saraswathi, R., 1993. Hydrobiology of two estuarine systems (Arasalar and Kaveri) of the southeast coast of India with special reference to plankton. Doctoral dissertation, Annamalai University, India, pp-178.

[47] Vaheeda, K. K. (2008) Ecological studies on the brackish waters of Kodungallur area, Kerala, India, Ph. D., thesis, Mahadma Gandhi University, 179 pp.

[48] Smita Achary, M.S., Sahu, G., Mohanty, A.K., Samatara, M.K., Panigrahy, S.N., Selvanayagam, M., Satpathy, K.K., Prasad, M.V.R. and Panigrahy, R.C., 2010. Phytoplankton abundance and diversity in the coastal waters of Kalpakkam, east coast of India in relation to the environmental variables. The Bioscan, 2, pp.553-568.

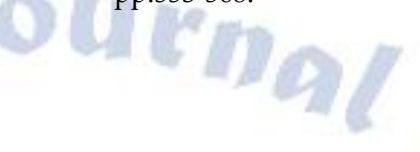

